\title{
Quartäre Klimaschwankungen: Schätzung der Temperaturschwankungen in Grönland mittels hochaufgelöster Isotopenmessung an im Eis archivierter Luft
}

\author{
Markus Leuenberger, Bern
}

\section{Einfiihrung}

Eisbohrkernmessungen sind insbesondere im Zusammenhang mit dem ansteigenden Treibhauseffekt besser bekannt geworden. Über die Analyse polarer Eisbohrkerne können die Konzentrationen der Treibhausgase $\mathrm{CO}_{2}, \mathrm{CH}_{4}$ und $\mathrm{N}_{2} \mathrm{O}$ in der Vergangenheit gemessen werden. Damit konnte gezeigt werden, dass über die Emission fossiler Brennstoffe diese Treibhausgase heute in einem Ausmass ansteigen, dass Anlass zur Sorge besteht (Intergovernmental Panel on Climate Change, IPCC, 2007; Siegenthaler et al. 2005). Die durch den verstärkten Treibhauseffekt - der prinzipiell ein lebensnotwendiger Effekt ist, da sonst auf der Erdoberfläche im Mittel eine Temperatur von $-15^{\circ} \mathrm{C}$ herrschen würde - steigende Temperatur führt unweigerlich zu einer klimatischen Veränderung. Hier sollte und muss eingegriffen werden mittels einer Beschränkung der $\mathrm{CO}_{2}$-Emission aus fossilen Brennstoffen.

Über Eismessungen können mannigfaltige Informationen gewonnen werden, sehr viele davon sind relevant, wenn es um Fragen zu Klimaveränderungen geht, da im Eis die Klimageschichte mehrerer hunderttausend Jahre archiviert ist. Deshalb erlauben solche Messungen, das Verständnis des Systems Erde retrospektiv zu erforschen: Warum gab es Eiszeiten, weshalb wurden diese Eiszeiten beendet, wodurch geschah dies? Warum war das Klima während den Eiszeiten so instabil? Wie gross waren die Temperaturveränderungen zwischen Eiszeiten und Zwischeneiszeiten beziehungsweise zwischen Warm- und Kaltphasen während der letzten Eiszeit? Dies sind Fragen, welche sich mittels Eisbohrkernen, zum Teil in Kombination mit anderen Klimaarchiven (Meeressedimente, Seesedimente, Baumringe und andere), erforschen lassen.

Eisbohrkerne haben aber weit mehr zu bieten als die oben erwähnten, äusserst nützlichen Konzentrationsmessungen. Physikalische Parameter des Eises, wie elektrische Leitfähigkeit, Staubgehalt und andere, erlauben durch Vergleich mit anderen ausgemessenen Eisbohrkernen die Erstellung einer vorläufigen Zeitskala, welche durch Folgeanalysen verfeinert wird. $\mathrm{Zu}$ diesen Folgeanalysen gehört die Bestimmung chemischer Spuren im Eis, welche Aufschluss über Emissionsstärke, Emissionsorte und Luftzirkulation chemi- scher Substanzen geben bzw. chemische Reaktionen erahnen lassen.

Dieser Beitrag wird jedoch insbesondere die Analysen stabiler Isotope an Eisbohrkernen in den Mittelpunkt stellen. Innerhalb eines Elementes gibt es normalerweise mehrere Isotope (Nuklide mit unterschiedlicher Neutronenzahl), welche entweder stabil oder radioaktiv sind. Beide Arten sind in der Forschung auch in der Eisbohrkernuntersuchung von grosser Bedeutung. Hier werden aber ausschliesslich stabile Isotope betrachtet. Es wird dabei der Frage nachgegangen, wie gross die Temperatursprünge während der letzten Eiszeit in Grönland waren.

\section{Stabile Isotope in Wassermolekülen als Thermometer}

Isotope sind Kerne eines Elementes, die sich in der Zahl ihrer Neutronen unterscheiden und somit unterschiedliche Massen besitzen. Dies wirkt sich unter anderem auf den Dampfdruck aus: Schwere Isotope haben einen kleineren Dampfdruck als leichte, d.h. sie kondensieren früher. Durch Wasserkondensation in Wolken und nachfolgendem Ausregnen beziehungsweise Ausschneien während des Transportes vom warmen Ozean zum kalten Eis verschiebt sich das Isotopenverhältnis des Wassers, und der Wasserdampf reichert sich in Sauerstoff-18 $\left({ }^{18} \mathrm{O}\right)$ oder im Deuterium (D) ab. Auf den Eisschilden werden daher sehr negative $\delta^{18} \mathrm{O}$ - und $\delta \mathrm{D}$-Werte gemessen (Abb. 1). Im Winter tritt dieser Effekt aufgrund des grösseren Temperaturgradienten zwischen Ozean und Eisschild stärker auf als im Sommer. $\delta$-Werte sind relative Abweichungen bezüglich eines Standards, für Wasser sind dies der so genannte SMOW-Wert (Standard Mean Ocean Water) und der SLAP-Wert (Standard Light Antarctic Precipitation). Da die Abweichungen relativ gering sind, werden sie mit 1000 multipliziert und man erhält die Promille-Einheit \%o.

Willi Dansgaard, ein Pionier der Eisanalysen, hat sich seit Mitte des letzten Jahrhunderts intensiv mit dieser Problematik auseinander gesetzt und dazu eine wegweisende Publikation veröffentlicht (DANSGAard 1964). Indem er die jährlich gemittelten $\delta^{18} \mathrm{O}$-Werte verschiedener Orte in Grönland gegen die mittleren Lufttemperaturen aufgetragen hat, ergab sich ein sehr enger Zusammenhang von $0,67 \% /{ }^{\circ} \mathrm{C}$ (Abb. 2). Man 


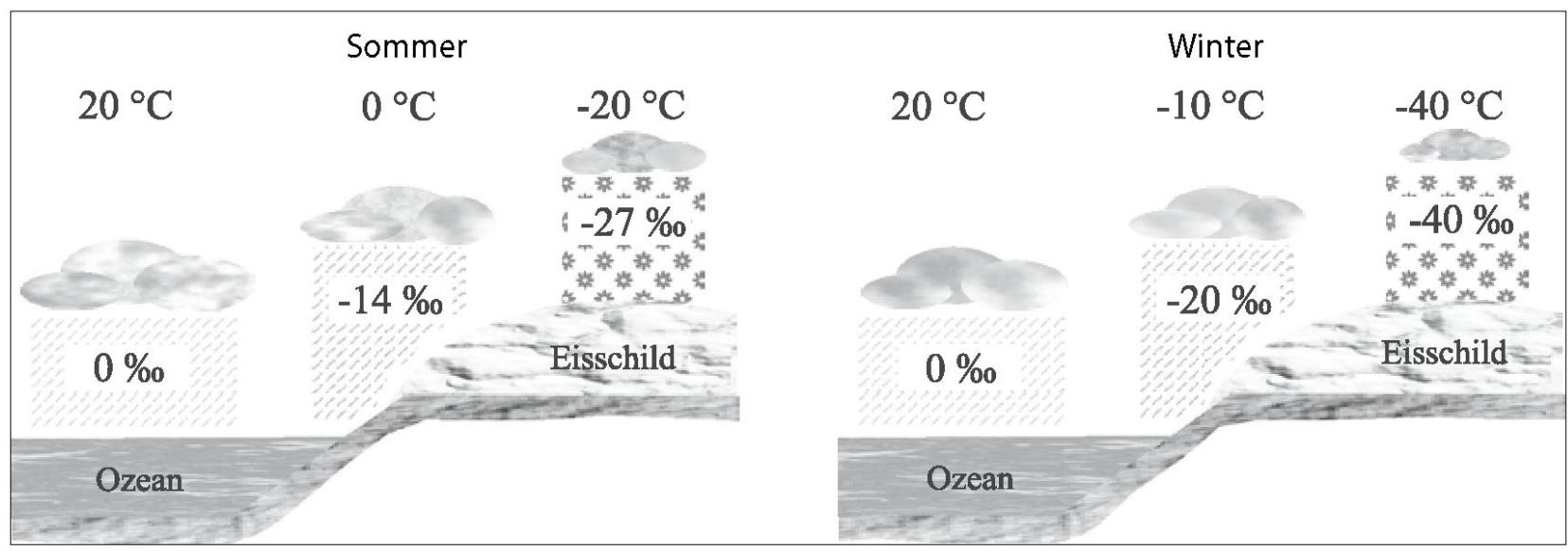

Abb.1: Abreicherung des Wassers in $\delta^{18} \mathrm{O}$ als Funktion der Temperatur und der Kondensationszyklen Isotope dependencies on temperature and condensation cycles Appauvrissement de l'eau en $\delta^{18} O$ comme fonction de la température et des cycles de condensation Graphik: J. ELSIG

nennt dies die räumliche oder geografische Abhängigkeit der Sauerstoffisotope von der Temperatur. Der Wert ist dabei abhängig vom Gebiet, das man untersucht, d.h. der Wert für Grönland kann nicht für die Antarktis verwendet werden. Durch diese räumliche Abhängigkeit war eine erste Ausgangslage geschaffen, wie man Variationen von stabilen Isotopen in Wassermolekülen interpretieren kann, welche man als Funktion der Tiefe in den Eisbohrkernen misst.

In den letzten 15 Jahren wurden in Grönland drei Tiefbohrungen durchgeführt: GRIP (Greenland Ice Core Project, Europa), GISP II (Greenland Ice Sheet Project II, USA) und NorthGRIP (multinational) Abbildung 3 stellt die Sauerstoffisotopenreihen von GRIP und NorthGRIP dar (NorthGRIP Members 2004). Die Tiefe des NorthGRIP-Kerns ist um ca. 30 Meter verschoben, sodass die Kurven übereinander zu liegen kommen. In den ersten $1500 \mathrm{~m}$ eines GRIP-Eisbohrkerns befindet man sich im Holozän, der heutigen Warmphase, bis $3000 \mathrm{~m}$ in der letzten Eiszeit und darunter in der vorangegangenen Warmzeit, dem so genannten Eem. Eine sehr hohe Übereinstimmung der beiden $\delta^{18} \mathrm{O}$-Kurvenverläufe ist deutlich erkennbar. Insbesondere die kurzen, schnellen Schwankungen in der letzten Eiszeit, die so genannten DansgaardOeschger-Ereignisse (DO-Ereignisse), stimmen sehr gut überein (DANSGAARD \& GUNDESTRUP 1993; GROOTES et al. 1993; JoHNSEN et al. 1992). Ebenso deutlich erkennbar sind die im Vergleich zu den GRIP-Werten negativeren NorthGRIP-Werte über die gesamte letzte Eiszeit hinweg (Abb. 3; NorthGRIP Members 2004). Dies könnte auf ein unterschiedliches Niederschlagseinzugsgebiet oder aber auf eine unterschiedli- che absolute Temperatur zurückzuführen sein. Dieser Problematik könnte mit dem Deuterium Exzess auf den Grund gegangen werden. Hier sollen allerdings nur die schnellen Schwankungen näher betrachtet werden.

Eine Möglichkeit der Interpretation der Daten besteht darin, die zeitlichen Variationen, welche im Eiskern als Funktion der Tiefe abgelegt sind, mit der oben erwähnten räumlichen Abhängigkeit direkt in Temperaturwerte umzusetzen. Auf diese Weise ergibt sich eine Temperaturveränderung in der Grössenordnung von $12^{\circ} \mathrm{C}$ zwischen Eiszeiten und Nacheiszeiten $\left(12{ }^{\circ} \mathrm{C}=8 \%\right.$ bei einem Wert von $0,67 \% /{ }^{\circ} \mathrm{C}$; vgl. $\mathrm{Abb}$. 2). Die DO-Variationen sind dabei fast so hoch wie der Übergang von der Eiszeit in die heutige Warmzeit. Es stellte sich daher die Frage, ob dies wirklich Temperaturvariationen sind oder ob es sich um Veränderungen eines anderen Parameters handelt. Wenn es tatsächlich Temperaturschwankungen sind, hat das Klima in der letzten Eiszeit verrückt gespielt. In den antarktischen Eisbohrkernen sind diese Kalt-Warmzeit-Zyklen viel weniger deutlich vorhanden, was den Schluss zulässt, dass diese schnellen Schwankungen mit Veränderungen in der Nordhemisphäre zu tun haben. Eine heute brennende Problematik stellt insbesondere die zeitliche Abfolge der Veränderungen in der Nordhemisphäre zu Veränderungen in der Südhemisphäre dar. Eine neue Studie weist mittels hochpräzisen Sauerstoff- zu Stickstoffverhältnismessungen im antarktischen Eis darauf hin, dass die nordhemisphärische Sommereinstrahlung die letzten vier Eiszeit/Nacheiszeit-Übergänge ausgelöst hat (KAWAMURA et al. 2007). 


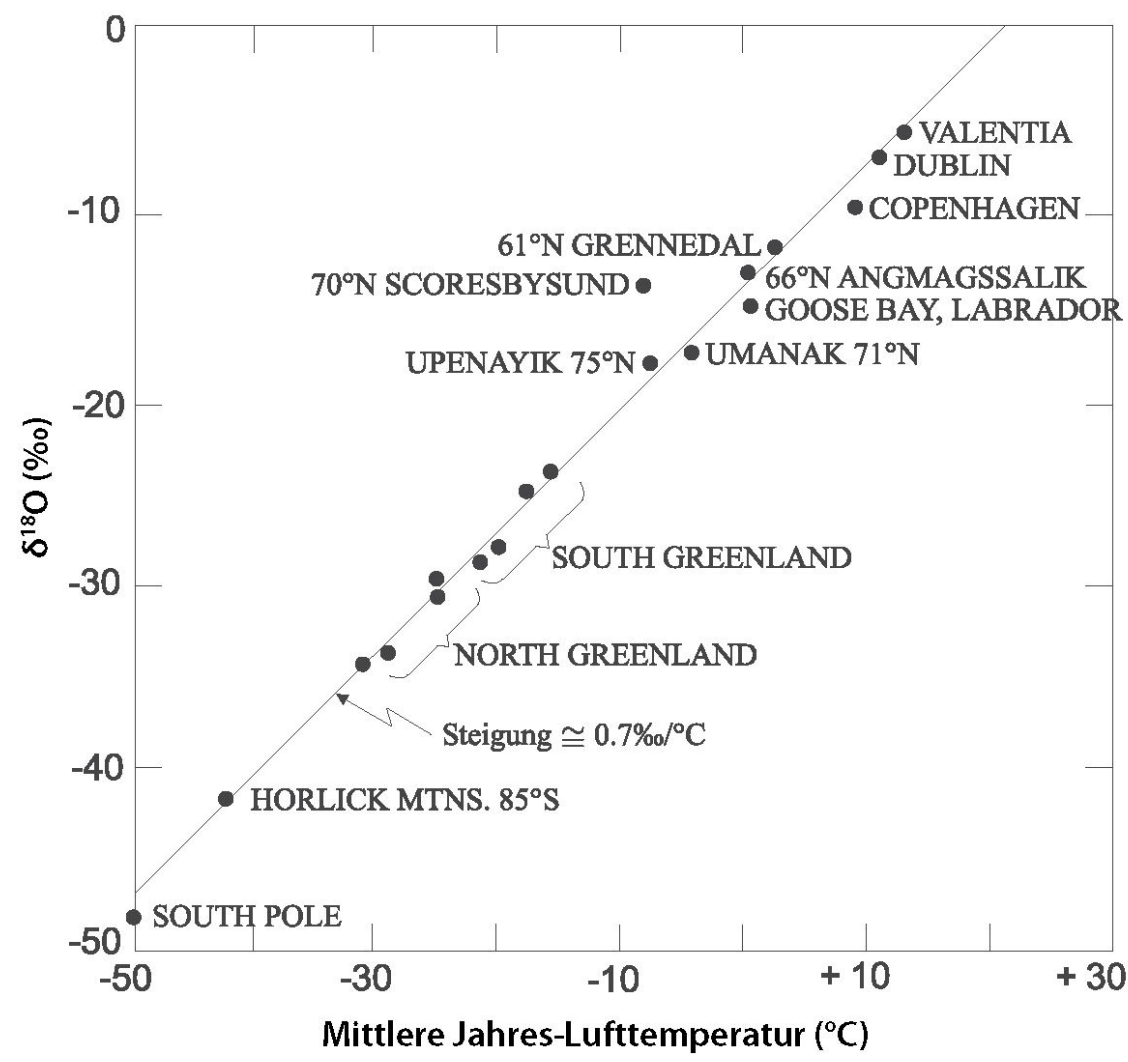

Abb. 2: Räumliche Abhängigkeit der Sauerstoffisotope $\left(\delta^{18} \mathrm{O}\right)$ von der Temperatur hauptsächlich für GrönlandStationen

Spatial dependence of oxygen isotopes $\left(\delta^{18} O\right)$ on temperature for Greenland stations mainly

Dépendance spatiale des isotopes d'oxygène $\left(\delta^{18} O\right)$ aux températures, principalement pour des stations du Groenland

Quelle: nach DANSGAARD 1964

\section{Widersprüche mit direkten, hochpräzisen Temperaturmessungen im Bohrloch}

Hochpräzise, auf $1 / 1000^{\circ} \mathrm{C}$ genaue Bohrloch-Temperaturmessungen als Funktion der Tiefe haben ergeben, dass die tatsächlich gemessene Temperaturkurve deutlich von der Kurve abweicht, welche für heutige Akkumulationsraten- und Temperaturbedingungen der GRIP-Station erwartet würde (DAHL-JENSEN et al. 1998). Mittels einer Entfaltungsrechnung des Temperatursignals als Funktion der Tiefe in zeitlich aufgetretenen Temperaturvariationen - man kann sich vorstellen, dass die im Eisbohrloch vorhandenen Temperaturvariationen sich mit der Zeit natürlich durch Diffusion ausgleichen - hat DAHL-JENSEN in Kopenhagen herausgefunden, dass zwischen der heutigen Warmzeit und der kältesten Phase der letzten Eiszeit in Grönland ein Temperaturunterschied von etwa $25^{\circ} \mathrm{C}$ vorhanden sein muss (DAHL-J ENSEN et al. 1998). Im Vergleich zu dem aufgrund der stabilen Isotope von Wasser abgeschätzten Temperaturunterschied von $12^{\circ} \mathrm{C}$ entspricht das einem Faktor 2. Das bedeutet erstens, dass die Umsetzung der Sauerstoffisotope in Temperaturwerte nicht so einfach ist, und zweitens, dass die von DANSGAARD gefundene räumliche Abhängigkeit nicht 1:1 in eine zeitliche Abhängigkeit umgesetzt werden kann. Bei einem gemessenen $\delta^{18} \mathrm{O}$ Unterschied von zirka $8 \%$ und einem Temperaturunterschied von $25^{\circ} \mathrm{C}$ ergibt sich eine Abhängigkeit von $0,32 \% /{ }^{\circ} \mathrm{C}$. Dies bedeutet, dass die zeitliche Abhängigkeit offenbar durch andere Faktoren als die räumliche Abhängigkeit bestimmt ist. Allerdings hat schon DansgaARD in seinen frühen Publikationen erwähnt, dass eine einfache Umsetzung der Sauerstoffisotopenwerte in Temperaturäquivalente gefährlich sein kann Dies hatte er aufgrund seiner breiten Erkenntnisse bezüglich der Einflussgrössen auf die Sauerstoffisotope des Wassers herausgefunden. Eine andere, unabhängige Methode zur Rekonstruktion der Temperaturen musste also gefunden werden. 


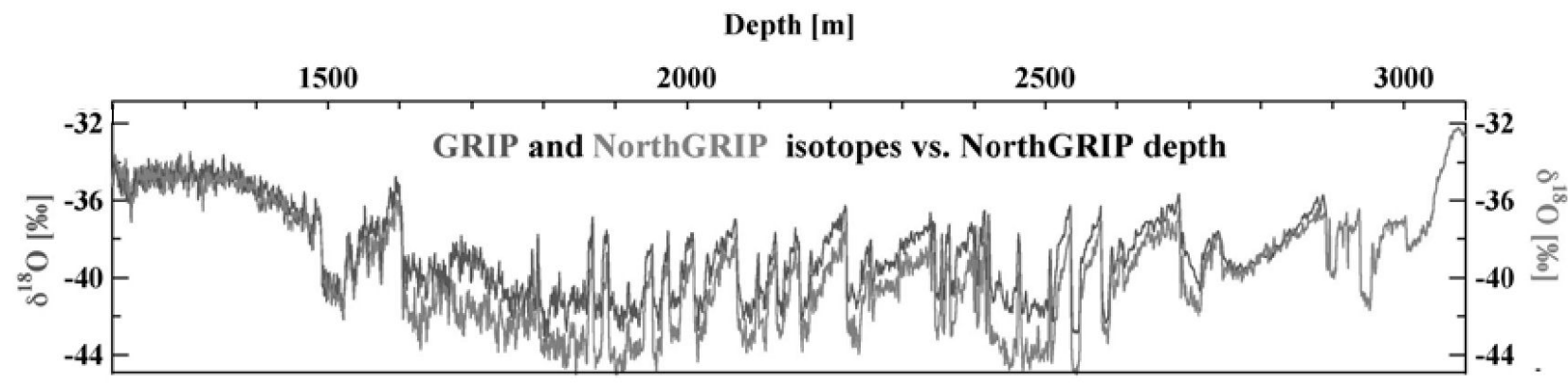

Abb. 3: Vergleich der Sauerstoffisotopenmessungen an GRIP- (dunkelgrau) und NorthGRIP-Eisbohrkernen (hellgrau) (NorthGRIP Members 2004). Bis 1500 m: Holozän, 1500-3000 m: letzte Eiszeit, ab 3000 m: Warmzeit (Eem).

Comparison of oxygen isotope measurements on GRIP (dark grey) and NorthGRIP-ice (light grey) (NoRTHGRIP MeMBers 2004). Holocene: until 1500 m, last glacial period: 1500-3000 m, last warm phase (Eem): below $3000 \mathrm{~m}$

Comparaison des enregistrements des isotopes d'oxygène du carottage GRIP (gris foncé) et NorthGRIP (gris clair) (NoRThGRIP Members 2004). Holocène: jusqu'à une profondeur de $1500 \mathrm{~m}$, dernière période glaciaire: 1500-3000 m, dernière période interglaciaire (Eem): au-dessous de $3000 \mathrm{~m}$.

\section{Stickstoffisotope als alternative Temperaturfühler}

Auf der Suche nach einem Ausweg aus diesem Dilemma ist man auf die Stickstoffisotope gekommen (Severinghaus \& Sowers 1995; Severinghaus et al. 1996). Stickstoff hat zwei stabile Isotope, ${ }^{14} \mathrm{~N}$ und ${ }^{15} \mathrm{~N}$. Das Verhältnis der beiden Isotope ist in der Atmosphäre sehr stabil, weil die Austauschflüsse im Vergleich zum Reservoir relativ gering sind. Dennoch gibt es ein Signal, welches mit der Temperatur zusammenhängt; um dies besser zu verstehen, müssen die Firnprozesse genauer betrachtet werden.

Im Firn lassen sich drei Zonen unterscheiden (Abb. 4; Sowers et al. 1992; Sowers et al. 1993):

(1) Die konvektive Zone, welche durch eine gute Durchmischung charakterisiert ist. Hier entspricht die Luftzusammensetzung derjenigen der Atmosphäre.

(2) Die diffusive Zone, in welcher der Transport der Luft durch molekulare Diffusion charakterisiert ist. Dies bedeutet, dass sich aufgrund der Erdanziehungskraft eine Entmischung der schweren und leichten Teilchen ergibt. Diese kann mit der Barometerformel quantifiziert werden. Die schweren Teilchen werden beim Firn/Eis-Übergang vermehrt vorkommen.

(3) Die nicht-diffusive Zone ist, wie der Name schon sagt, durch eine gegen Null strebende Diffusionskonstante charakterisiert. Hier wird die Luft in ihrer Zusammensetzung, obwohl noch nicht eingeschlossen, schon konserviert. Allerdings weisen neueste Ergebnisse auf eine zusätzliche Verände- rung der Luftzusammensetzung beim Abschlussprozess der Blasen hin (HuBER et al. 2006a).

Die entscheidende Zone ist somit die zweite Zone, in der sich physikalische Prozesse abspielen. In dieser Zone führt ein weiterer Effekt, die Thermodiffusion, zu einer zusätzlichen Anreicherung der schweren Teilchen im unteren Teil der Zone, wenn sich die Oberfläche abrupt erwärmt. Kommt es dagegen zu einer abrupten Abkühlung, wird die gravitative Anreicherung vermindert.

Beide Phänomene entsprechen geringen Verschiebungen der isotopischen Zusammensetzung. Die gravitative Anreicherung beträgt für verschiedene Firnkonditionen (Stationen) 0,2-0,5\% (grösserer Wert für Stationen mit tieferer Abschlusszone, welche kälteren Konditionen mit geringeren Akkumulationsraten entsprechen). Die Thermodiffusion ist noch weniger ausgeprägt und oftmals nur dann signifikant ausmessbar, wenn es zu grossen abrupten Temperaturveränderungen kommt. Die Verschiebungen aufgrund solcher Temperatursprünge entsprechen ca. der Hälfte des gravitativen Effektes. Diesen beiden Prozessen überlagert ist im oberen Teil der diffusiven Zone ein saisonaler Thermodiffusionseffekt aufgrund der sich jahreszeitlich ändernden Temperatur. Dieses Signal geht allerdings mit der Tiefe wieder verloren (Diffusion löscht das Signal aus) und ist somit nicht in den Gasblasen konserviert. Es kann allerdings mit direkter Firngasbeprobung ausgemessen werden. Um solch kleine Signale signifikant zu bestimmen, muss auf Hundertstel Promille genau gemessen werden 


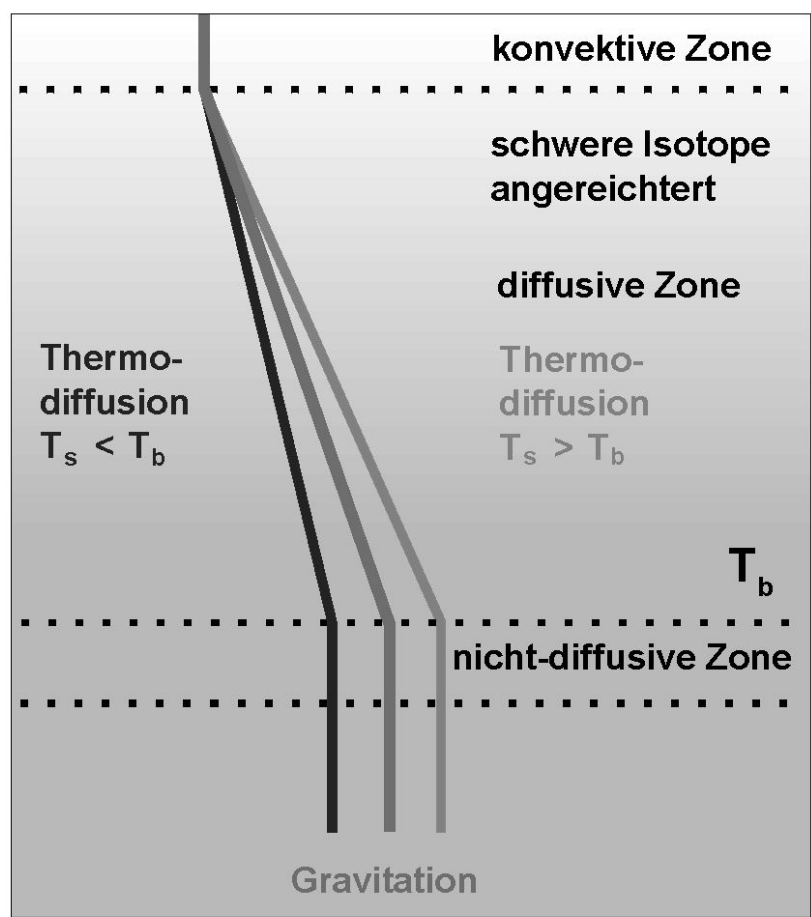

Abb 4: Einteilung des Firns

In der gut durchmischten konvektiven Zone (oben) entspricht die Zusammensetzung der Luft derjenigen der Atmosphäre. In der diffusen Zone (Mitte) wird die Zusammensetzung durch die Erdanziehungskraft verändert. Aufgrund der Thermodiffusion ergibt sich je nach Temperaturereignis an der Oberfläche eine erhöhte Anreicherung der schweren Teilchen (Oberfläche erwärmt sich, $T_{t}>T_{b}$ ), oder es kommt zu einer Verminderung der gravitativen Anreicherung (Oberfläche kühlt sich $\mathrm{ab}, \mathrm{T}_{\mathrm{t}}<\mathrm{T}_{\mathrm{b}}$ ). In der nicht-diffusiven Zone (unten) geht die Diffusionskonstante gegen Null.

Classification of firn sections

Classification des sections du névé

können. Dies geschieht mittels Isotopenmassenspektrometrie. Bei dem verwendeten Massenspektrometer lassen sich acht Masse/Ladungs-Verhältnisse mit sehr hohen Genauigkeiten simultan aufzeichnen. In einem Vorgerät wird das Eis geschmolzen, dann wird die Luft vom Wasser entkoppelt und dem Massenspektrometer zugeführt.

Diese Methodik wurde erfolgreich an NorthGRIP-Eis angewandt (HuBer et al. 2006b), und die DO-Ereignisse 8-17 wurden mit ihr ausgemessen. Die Ergebnisse sind in Abbildung 5 zusammengefasst und interpretiert. Die $\delta^{15} \mathrm{~N}$-Werte zeigen eine sehr gute Gleichläufigkeit mit den $\delta^{18} \mathrm{O}$-Messungen des Eiswassers. Die mittels Firnverdichtungsmodell und Wärmediffusionsmodell berechnete und an die $\delta^{15} \mathrm{~N}$-Werte angepasste Temperatur ist in der unteren Teilgrafik zu sehen. Es ist $\mathrm{zu}$ erkennen, dass die Temperatursprünge bei den DOEreignissen zwischen 8 und $15^{\circ} \mathrm{C}$ betragen. Dies sind enorm hohe Temperatursprünge, welche mit grossen Veränderungen des Erdklimasystems einhergehen.

\section{Implikationen fuir die stabilen Isotope in Wassermolekülen}

Trägt man die $\delta^{18} \mathrm{O}$-Werte des Eiswassers gegen die mittlere, aus den $\delta^{15} \mathrm{~N}$-Werten ermittelte Temperatur an den jeweils entsprechenden Orten auf, so erhält man eine gute Korrelation mit einer Steigung von $0,37 \% /{ }^{\circ} \mathrm{C}$. Im Gegensatz zum Wert von $0,67 \% /{ }^{\circ} \mathrm{C}$, den DANsGaARD (1964) für eine räumliche Abhängigkeit gefunden hat (Abb. 6), entspricht dieser Wert von $0,37 \% /{ }^{\circ} \mathrm{C}$ einer zeitlichen Abhängigkeit, wobei Daten über mehrere Tausend Jahre verwendet worden sind (Cuffey et al. 1995; Johnsen et al. 1995). Grundsätzlich können zwei Erklärungsansätze herangezogen werden:

(1) Eine Veränderung der Quellentemperatur (Abb. 6, links): Es ist zu erwarten, dass beim Übergang von der letzten Eiszeit ins heutige Holozän eine Quellentemperaturveränderung stattgefunden hat. Dies wurde in der Grafik durch eine Verschiebung der Dansgaard-Abhängigkeit durch den Mittelpunkt (graues Rhomboid) der Messwerte im Vergleich zu den heutigen Konditionen berücksichtigt. Durch die Pfeile ist somit eine zusätzliche Veränderung angetönt, welche die vom räumlichen Zusammenhang abweichenden Werte erklärt. Die Warmphasen können, im Vergleich zur mittleren eiszeit-/nacheiszeitlichen Temperaturveränderung des Oberflächenozean-Quellengebietes, durch eine kleinere Erhöhung der Quellentemperatur, die Kaltphasen durch eine erhöhte Quellentemperaturabsenkung erklärt werden.

(2) Eine Veränderung der jahreszeitlichen Verteilung des Niederschlags (Abb. 6, rechts): Ebenso könnten die Warmphasen durch mehr Winterniederschlag und die Kaltphasen durch weniger Winterniederschlag erklärt werden.

Welcher dieser beiden Erklärungsansätze korrekt ist kann zurzeit noch nicht schlüssig beantwortet werden, wobei jedoch eher eine Veränderung der Niederschlagsverteilung infrage kommt.

\section{Fazit}

Zusammenfassend lässt sich sagen, dass stabile Isotope in Wassermolekülen einerseits durch die Temperatur, andererseits durch die Verteilung des Niederschlags über das Jahr beeinflusst werden. Dies konnte insbesondere durch direkte Bohrlochtemperaturbe- 


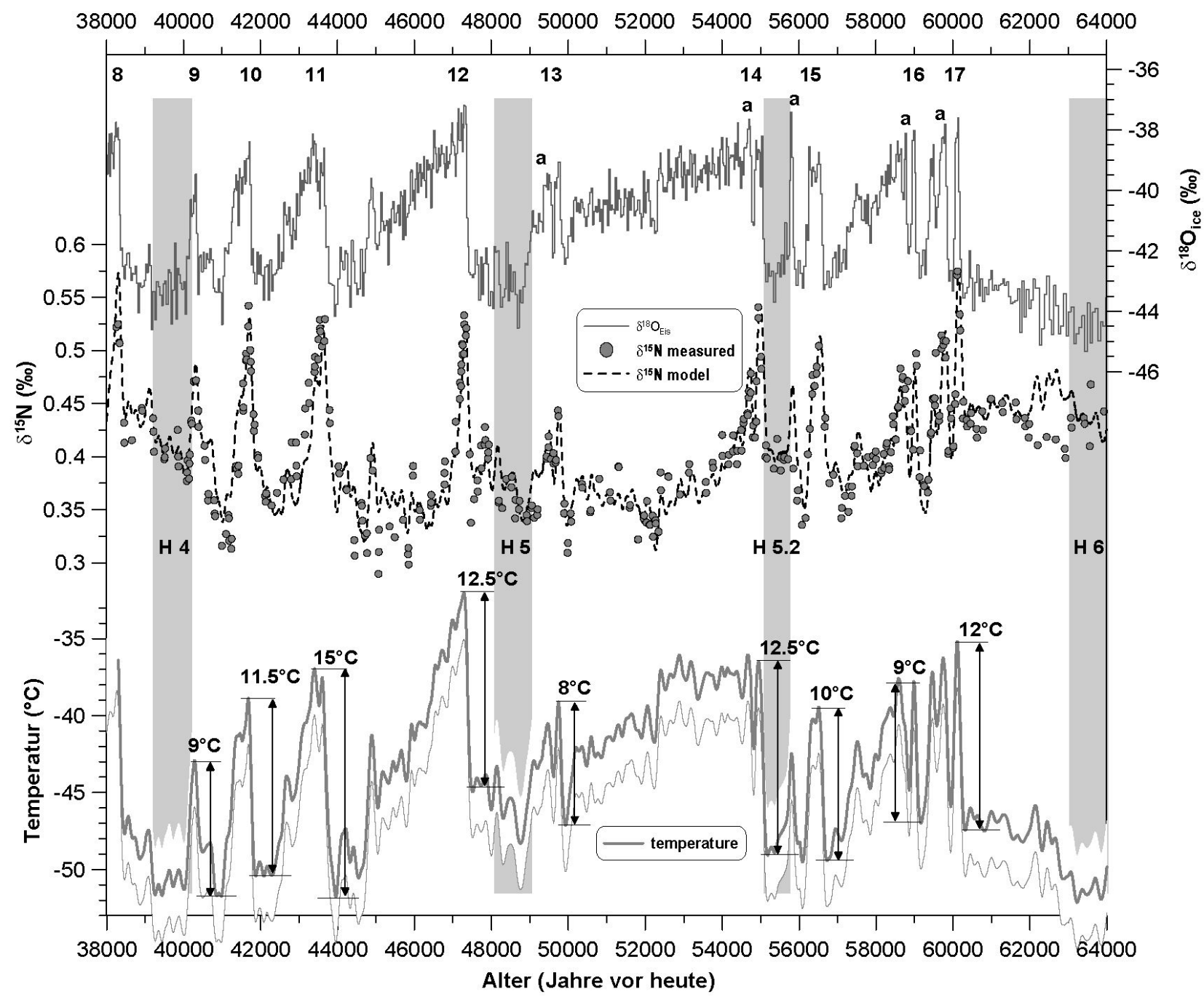

Abb. 5: NorthGRIP $\delta^{18} \mathrm{O}_{\text {ice }}$ (oben; NorThGRIP Members 2004), NorthGRIP $\delta^{15} \mathrm{~N}$ (Mitte) sowie Oberflächentemperatur (graue Linie, unten) für die Zeitperiode der DO-Ereignisse 8-17

Alle Kurven sind auf der Altersskala GRIP2001/ss09sea (JohNSEN et al. 2001; NoRThGRIP MEMBERs 2004) wiedergegeben. $\delta^{15} \mathrm{~N}$-Messresultate (graue Punkte) sind mit der Modellkurve verglichen (gestrichelte Linie). Die Temperaturkurve ist mittels des Firnverdichtungs- und des Wärmediffusionsmodells (SchwaNDER et al. 1997) an die Messwerte angepasst. Die Temperatur ist als geglättete Kurve (50 Jahre, mit einem Fehler von $\pm 3^{\circ} \mathrm{C}$, grau gepunkteter Bereich unten) gezeichnet. Temperatursprünge sind für alle untersuchten DO-Ereignisse angegeben ausser für DO 8, da dieses Ereignis nicht vollständig vermessen wurde. Heinrich (H)-Ereignisse sind grau hinterlegt.

NorthGRIP $\delta^{18} O_{\text {ice }}$ (top; NorThGRIP Members 2004), NorthGRIP $\delta^{15} N$ (middle) and surface temperature estimates (grey line, bottom) for the time period of DO-events 8-17

NorthGRIP $\delta^{18} O_{\text {ice }}$ (en haut; NoRThGRIP MEMBERs 2004), North GRIP $\delta^{15} N$ (au milieu) et température de surface (ligne grise en bas) pour la période de temps des événements DO 8-17

stimmungen herausgefunden werden. Die dadurch erhaltene Unstimmigkeit der Sauerstoffisotopenvariationen bezüglich der Temperatur zwischen der räumlichen und der zeitlichen Abhängigkeit führte zur Suche nach einer neuen Temperaturrekonstruktionsmethodik. Diese wurde in der Veränderung der Isotopenge- halte mittels der Thermodiffusion gefunden. Das $\delta^{15} \mathrm{~N}$ kann als sehr wichtiger und zuverlässiger Temperaturindikator herangezogen werden, insbesondere für schnelle, abrupte Temperatursprünge, z.B. von $10-15^{\circ} \mathrm{C}$ innerhalb weniger Dekaden, wie dies am Beispiel von NorthGRIP sehr schön gezeigt wurde. 

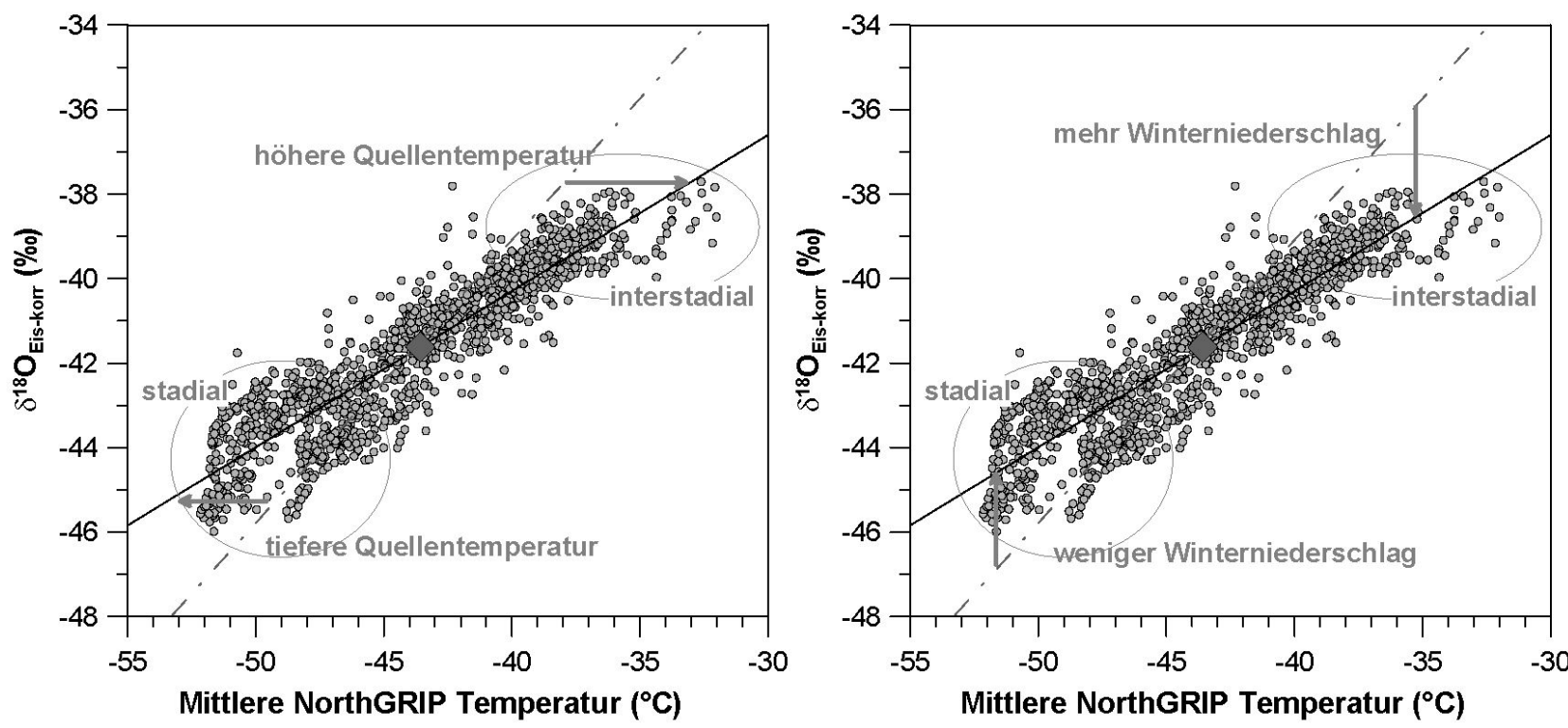

Abb. 6: Mittels $\delta^{18} \mathrm{O}$ von Meeressedimenten korrigierte $\delta^{18} \mathrm{O}$-Werte des Eiswassers (NorthGRIP) als Funktion der mittleren, aus $\delta^{15} \mathrm{~N}$-Werten rekonstruierten Stationstemperatur (graue Punkte); graues Rhomboid: Mittelwerte der beiden Parameter

Die Korrelation ergibt eine mittlere Steigung von $0,37 \% /{ }^{\circ} \mathrm{C}$ (schwarze Linie), welche deutlich unter derjenigen der räumlichen Korrelation nach DANSGAARD $\left(0,67 \% /{ }^{\circ} \mathrm{C}\right.$, Punktstrich-Linie) liegt, jedoch in guter Übereinstimmung mit der gefundenen Beziehung zur Bohrlochtemperatur steht. Als Erklärungsansätze für die Abweichung der Geraden können eine Quellentemperaturveränderung (links) und die jahreszeitliche Niederschlagsverteilung (rechts) herangezogen werden. Stadial: Kaltphase; interstadial: Warmphase; horizontale Pfeile (linke Grafik): zusätzliche Quellentemperaturänderung gegenüber der mittleren eiszeit-/nacheiszeitlichen Temperaturverschiebung; vertikale Pfeile (rechte Grafik):mögliche Verschiebung der jahreszeitlichen Niederschlagsverteilung, welche sich nach den in Abb. 1 dargestellten Prozessen auf die $\delta^{18} \mathrm{O}-$ Werte auswirkt.

Dependence of $\delta^{18} \mathrm{O}$-values of ice water (NorthGRIP), corrected for source temperature effects based on ocean $\delta^{18} \mathrm{O}$-values, on local temperature estimates retrieved from $\delta^{15} \mathrm{~N}$-values (grey dots); grey rhomboid: mean of these values

Valeurs $\delta^{18} O$ de l'eau de glace, corrigées à l'aide des valeurs $\delta^{18} O$ des sédiments de mer comme fonction des valeurs moyennes de températures reconstruites à partir des valeurs $\delta^{15} N$ (points gris); rhomboïde gris: valeurs moyennes des deux parametres

\section{Dank}

Diese Arbeit ist Teil des NorthGRIP Ice Core Project des Departements Geophysik am Niels-Bohr-Institut für Astronomie, Physik und Geophysik, Universität Kopenhagen, unterstützt durch Forschungsfinanzierung in Dänemark (SNF), Belgien (FNRS-CFB), Frankreich (IFRTP und INSU/CNRS), Deutschland (AWI), Island (RannIs), Japan (MEXT), Schweden (SPRS), der Schweiz (SNF) und den Vereinigten Staaten (NSF). Die Studie ist ebenfalls Teil des European Project of Ice Coring in Antarctica (EPICA), eines gemeinsamen Forschungsprojekts der European Science Foundation und der European Commission, finanziert durch die Europäische Union (EPICA-MIS) und durch staatliche Beiträge aus Belgien, Dänemark, Frankreich, Deutschland, Italien, den Niederlanden, Norwegen, Schweden, der Schweiz und Grossbritannien.

\section{Literatur}

Cuffey, K.M., Clow, G.D., Alley, R.B., Stuiver, M., Waddington, E.D. \& R.W. Saltus (1995): Large arctic temperature change at the Wisconsin-Holocene glacial transition. - In: Science 270 455-458.

Dahl-Jensen, D., Mosegaard, K., Gundestrup, N., Clow, G.D., Johnsen, S.J., Hansen, A.W. \& N. Balling (1998): Past temperatures directly from the Greenland Ice Sheet. - In: Science 282: 268-271.

DansgaARD, W. (1964): Stable isotopes in precipitation. - In: Tellus 16: 436-468.

DansgaARd, W. \& N. Gundestrup (1993): Greenland: a temptation and a challenge. - In: Endeavour, New Series 17:12-16.

Grootes, P.M., Stuiver, M., White, J.W.C., Johnsen, S. \& J. Jouzel (1993): Comparison of oxygen isotope 
records from the GISP2 and GRIP Greenland ice cores. - In: Nature 366: 552-554.

Huber, C., Beyerle, U., Leuenberger, M., SchwanDer, J., Kipfer, R., Spahni, R., Severinghaus, J.P. \& K. WEILER (2006a): Evidence for molecular size dependent gas fractionation in firn air derived from noble gases, oxygen, and nitrogen measurements. - In: Earth and Planetary Science Letters 243: 61-73.

Huber, C., Leuenberger, M., Spahni, R., Flǘckiger, J., Schwander, J., Stocker, T.F., Johnsen, S., Landals, A. \& J. Jouzel (2006b): Isotope calibrated Greenland temperature record over Marine Isotope Stage 3 and its relation to CH4. - In: Earth and Planetary Science Letters 243: 504-519.

Intergovernmental Panel on Climate Change (IPCC), Solomon, S., Qin, D., Manning, M., Marquis, M., Averyt, K., Tignor, M.M.B., Miller, Jr., H.L. \& Z. Chen (eds) (2007): Climate change 2007. The physical science basis. - Contribution of Working Group 1 to the Fourth Assessment Report of the Intergovernmental Panel on Climate Change. - Cambridge: Cambridge University Press, http://ipcc-wg1.ucar.edu/wg1/ wg1-report.html.

Johnsen, S.J., Clausen, H.B., Dansgaard, W., Fuhrer, K., Gundestrup, N., HAmmer, C.U., Iversen, P., Jouzel, J., STAuffer, B. \& J.P. StefFEnSEN (1992): Irregular glacial interstadials recorded in a new Greenland ice core. - In: Nature 359: 311-313.

Johnsen, S.J., Clausen, H.B., Dansgaard, W., GundeStruP, N.S., Hammer, C.U. \& H. TAuber (1995):The Eem stable isotope record along the GRIP ice core and its interpretation. - In: Quaternary Research 43: 117-124. Johnsen, S.J., Dahl-JENSEN, D., Gundestrup, N., STEFfensen, J.P., Clausen, H.B., Miller, H., Masson-Delmotte, V., SveinbJöRnsdottiR, A.E. \& J. White (2001) Oxygen isotope and palaeotemperature records from six Greenland ice-core stations: Camp Century, Dye 3 GRIP, GISP2, Renland and NorthGRIP. - In: Journal of Quaternary Science 16:299-307.

Kawamura, K., Parrenin, F., Lisiecki, L., Uemura, R., Vimeux, F., Severinghaus, J.P., Hutterli, M.A., NAKAzawa, T., Aoki, S., Jouzel, J., Raymo, M.E., Matsumoto, K., Nakata, H., Motoyama, H., Funta, S., GotoAzuma, K., Fuji, Y. \& O. Watanabe (2007): Northern Hemisphere forcing of climatic cycles in Antarctica over the past 360,000 years. - In: Nature 448: 912-916. NorTHGRIP MEMBERs (2004): High resolution climate record of the Northern Hemisphere reaching into the last glacial interglacial period. - In: Nature 431:147-151. Schwander, J., Sowers, T., Barnola, J.-M., Blunier, T., Malaizé, B. \& T. Fuchs (1997): Age scale of the air in the summit ice: Implication for glacial-interglacial temperature change. - In: Journal of Geophysical Research 102: 19483-19494.

Severinghaus, J. \& T. Sowers (1995): Thermal diffusion as a temperature-change indicator in ice core climate records (abstract). - In: Eos, Transactions, Ame- rican Geophysical Union (AGU) 76 (46), Fall Meeting, Supplement, F291.

Severinghaus, J.P., Brook, E.J., Sowers, T. \& R.B. Alley (1996): Gaseous thermal diffusion as a gasphase stratigraphic marker of abrupt warmings in ice core climate records (abstract). - In: Eos, Transactions, American Geophysical Union (AGU) 77 (17), Spring Meeting, Supplement, S157.

Siegenthaler, U., Stocker, T.F., Monnin, D., LÜthi, D., Schwander, J., Stauffer, B., Raynaud, D., Barnola J.-M., Fischer, H., Masson-Delmotte, V. \& J. Jouzel (2005): Stable carbon cycle-climate relationship during the late Pleistocene. - In: Science 310: 1313-1317.

Sowers, T., Bender, M., Raynaud, D. \& Y.S. KorotKEVICH (1992): $\delta^{15} \mathrm{~N}$ of $\mathrm{N}_{2}$ in air trapped in polar ice: $\mathrm{A}$ tracer of gas transport in the firn and a possible constraint on ice age-gas age differences. - In: Journal of Geophysical Research 97: 15683-15697.

Sowers, T., Bender, M., Labeyrie, L., Martinson, D., Raynaud, D., Pichon, J.J. \& Y.S. Korotkevich (1993) A 135.000-year Vostok-Specmap common temporal framework. - In: Paleoceanography 8: 737-766.

\section{Zusammenfassung: Quartäre Klimaschwankungen: Schätzung der Temperaturschwankungen in Grön- land mittels hochaufgelöster Isotopenmessung an im Eis archivierter Luft}

Eisbohrkerne bieten einen Zugang zu klimatischer Information, welche zeitlich gut geordnet und in hoher Präzision ausgemessen werden kann. Stabile Isotope in Wassermolekülen können relativ einfach mit hoher Genauigkeit gemessen werden und geben einen ersten Eindruck, wie sich die lokale Temperatur zeitlich verändert hat. Allerdings dürfen die Isotopenvariationen des Wassers nicht 1:1 in lokale Temperaturen umgesetzt werden, da es neben der Temperatur auch noch andere Einflussgrössen gibt, wie die Quellentemperatur und die jahreszeitliche Niederschlagsverteilung. Variationen von Stickstoffisotopen aufgrund der Thermodiffusion erweisen sich als sehr guter Temperatur-Proxy für grosse und schnelle Temperatursprünge, wie dies die Dansgaard-Oeschger-Ereignisse darstellen. An NorthGRIP-Eis wurden mit dieser neuen Methodik Temperatursprünge zwischen 8 und $15^{\circ} \mathrm{C}$ für die DansgaardOeschger-Ereignisse 8-17 gemessen. Die Genauigkeit dieser Temperatursprünge beträgt ca. $3^{\circ} \mathrm{C}$.

Schlüsselwörter: abrupte Klimaveränderung, Stickstoffisotopenverhältnisse, Eisbohrkern-Messungen, Quartär

\section{Abstract: Quaternary climate change: Estimate of temperature change in Greenland by means of highly resolved isotope measurements of air enclosed in ice}

Ice cores carry a wealth of climatic information which are temporally well constraint. Stable isotope ratios in water can be measured relatively accurately and easily, 
yielding a good first impression of past changes of the local temperature. However, caution should be exercised in inferring local temperatures from water isotope ratios because of other parameters that influence isotopic composition, such as source temperature and seasonal distribution of precipitation. In situations with rapid and abrupt temperature change, as was recorded for the Dansgaard-Oeschger events, nitrogen isotope ratios may prove to be very useful because of their link to gravitation enrichment and thermal diffusion. Measurements made with this new proxy on NorthGRIP ice revealed abrupt temperature shifts of $8-15^{\circ}$ Celsius for the Dansgaard-Oeschger events 8-17. Accuracy of the measurements lies at about 3 degrees.

Keywords: abrupt climate change, nitrogen isotope ratio, ice core measurement, Quaternary

\section{Résumé: Variations climatiques quaternaires: recons- truction des variations de température au Groenland à partir d'une analyse isotopique à haute résolution appliquée aux bulles d'air contenues dans la glace}

Les carottes de glace donnent accès à des informations climatiques passées. Ces informations sont bien ordonnées dans le temps et peuvent être enregistrées avec une haute précision. De façon relativement simple, on peut analyser précisément les proportions relatives des isotopes stables dans les molécules d'eau. Ces enregistrements offrent une première impression des variations temporelles de la température locale au Groenland. Toutefois, les variations isotopiques enregistrées ne peuvent pas être interprétées directement comme des variations de la température locale. En effet, d'autres facteurs influencent la signature isotopique dans l'eau: par exemple la température de source et la répartition saisonnière des précipitations. Les variations des isotopes d'azote qui se produisent en raison de la thermodiffusion s'avèrent être de très bons indicateurs de température pour des sauts de température importants et rapides comme dans le cas des événements Dansgaard-Oeschger. Appliquée à la glace du carottage NorthGRIP, cette nouvelle méthodologie a permis d'enregistrer des sauts de température entre 8 et $15^{\circ} \mathrm{C}$ pour les événements Dansgaard-Oeschger 8-17. La précision des sauts de température est d'environ $3^{\circ} \mathrm{C}$.

Mots-clés: changement climatique abrupt, rapport isotopique de l'azote, mesures de carottes de glace, Quaternaire

Prof. Dr. Markus Leuenberger, Physikalisches Institut, Universität Bern, Sidlerstrasse 5, CH-3012 Bern, Schweiz, und Oeschger Centre for Climate Change Research, Universität Bern, Erlachstrasse 9a, CH3012 Bern, Schweiz.

e-mail: leuenberger@climate.unibe.ch

Manuskripteingang/received/manuscrit entré le 29.5.2008

Annahme zum Druck/accepted for publication/accepté pour l'impression: 4.9 .2008 